\title{
On a Dual Direct Cosine Simplex Type Algorithm and Its Computational Behavior
}

\author{
E. M. Badr ${ }^{1}$ and Khalid Aloufi ${ }^{2}$ \\ ${ }^{1}$ Scientific Computing Department, Faculty of Computers \& Artificial Intelligence, Benha \\ University, Benha, Egypt. badrgraph@gmail.com \\ ${ }^{2}$ College of Computer Science \& Engineering Taibah University Saudi Arabia \\ koufi@taibahu.edu.sa
}

\begin{abstract}
The goal of this paper is to propose a dual version of the direct cosine simplex algorithm (DDCA) for general linear problems. Unlike the two-phase and the big-M methods, our technique does not involve artificial variables. Our technique solves the dual Klee-Minty problem in two iterations and solves the dual Clausen's problem in four iterations. The utility of the proposed method is evident from the extensive computational results on test problems adapted from NETLIB. Preliminary results indicate that this dual direct cosine simplex algorithm (DDCA) reduces the number of iterations of two-phase method.
\end{abstract}

Keywords: linear programming; dual simplex method; dual direct cosine method; two-phase method.

\section{Introduction:}

Linear programming is an important cornerstone in the optimization theory. Many realistic problems can be formulated by means of linear mathematical models. The simplex algorithm is the most used tool for solving linear programs. It is an iterative method that was developed by Dantzig $[1,2,3]$.

There are many pivot rules for the simplex type algorithm like exterior point simplex algorithm [4, 5, 6] and max-out-in pivot rule [7]. It is known that the application of the simplex algorithm requires at least one basic feasible solution. The two-phase and big-M methods are the most familiar technique for the research of an initial feasible basis. The main drawback of these techniques is requiring the introduction of artificial variables, increasing the dimension of the problem. Wei-Chang Yeh and H.W. Corley [8] proposed a simple direct cosine simplex algorithm (DCA) which solves the KleeMinty Problem [9] in two iterations and reduced the number of iterations of Simplex 
in most cases in their computational experiment. In this paper, we propose a dual version of a simple direct cosine simplex algorithm (DDCA) which solves the dual Klee-Minty class of problem in two iterations while the Two phases method solves this class in $n+1$ iterations where $n$ is the size of the problem. Our technique also solves Clauser class of problems in four iterations but the two phase method solves this class in $2 n-1$ iterations where $n$ is the size of the problem. Our technique does not require the introduction of artificial variables.

The rest of the paper is organized as follows. Section 2 describes the proposed DDCA algorithm and its characteristics. Benchmark problems "Klee-Minty and Clausen problems" are presented in Section 3. In Section 4, we introduce illustrations of the proposed algorithm with help of two examples. Computational experiments are presented in Section 5, followed by concluding remarks and directions of future research in Section 6.

\section{Dual Cosine Simplex Algorithm (DDCA).}

We consider the linear programming (LP) problem in standard form:

$$
\max \left\{b^{T} y: A^{T} y=c ; y \geq 0\right\}, \text { where } A \text { is an } m \times n \text { matrix, } x \text { and } c \text { are }
$$

$n$-dimensional vectors and $T$ denotes transposition. The dual of $(\mathrm{P})$ is the problem

$$
\min \left\{c^{T} x: A x \geq b\right\} \text { where } y \text { is an } m \text {-dimensional vector. }
$$

For constraint $i$ of (D), define $\cos \theta_{i}=\left(\sum_{j \in N} a_{i j} c_{j}\right)^{2} / \sum_{j \in N}\left(a_{i j}\right)^{2}$ as the cosine of angle $\theta_{i}$ between the constraints $i$ and the objective function where $b_{i}<0$ and $N$ is the index set of the non-basic variables.

Remark: The above cosine criterion is only a simple observation without any further proof. Hence, the cosine criterion is not always true.

\section{Dual Cosine Simplex Method (DCSM).}

Require: infeasible basis

While $b_{i}<0$

Step 1: (Dual feasibility Condition). Let $N$ is the index set of the non-basic variables. The leaving variable,$x_{i}$, is the basic variable having the maximum $\cos \theta_{i}$ for minimization problem, where

$$
\cos \theta_{i}=\left(\sum_{j \in N} a_{i j} c_{j}\right)^{2} / \sum_{j \in N}\left(a_{i j}\right)^{2} \text { is }
$$


the angle between the constraint $i$ and the objective function. If there is a tie, then choose the variable with the most negative value in right hand side.

Step 2: (Dual optimality condition). Given that, $x_{i}$, is the leaving variable, the entering variable is the non-basic variable $a_{i j}<0$ that corresponds to

$$
\min \left\{\left|\frac{b_{i}}{a_{i j}}\right|: a_{i j}<0 \text { and } j \in N\right\}
$$

The ties are broken arbitrary. If $a_{i j} \geq 0$ for all non-basic variables then the problem has no feasible solution.

\section{End while}

Step 3: Apply a pivoting

The current basis is feasible

Apply the simplex algorithm.

\section{Benchmark problems}

In this Section we present two well-known classes of linear programming problems, Klee-Minty class of problems [10] is the first problem and the other is Clausen class of problems [11] as illustrated in the following models:

$$
\begin{array}{clll}
\max & \sum_{j=1}^{n} 10^{n-j} x_{j} & \max & \sum_{j=1}^{n}(4 / 5)^{j} x_{j} \\
& & x_{1} \leq 1 \\
\text { subject to } & 2 \sum_{j=1}^{i-1} 10^{i-j} x_{j}+x_{i} \leq 100^{i-1} & \text { subject to } & 2 \sum_{j=1}^{i-1}(5 / 4)^{i-j} x_{j}+x_{i} \leq 5^{i-1} \\
& x_{j} \geq 0, \quad i=1,2, \ldots n & & x_{j} \geq 0, \quad i=2, \ldots n \\
\text { Klee-Minty problem } & \text { Clausen problem }
\end{array}
$$

Klee and Minty $[10,12]$ were the first to prove that Simplex has exponential worst-case running time in 1972. An interesting result is that the dual simplex method solves the Klee-Minty problem in a polynomial number of iterations [11]. A more challenging exponential example is given by Clausen $[10,11]$. The main feature of Clausen's example is that the primal simplex method is exponential on the primal problem while the dual simplex is exponential on the dual problem.

The following examples show the superiority of our technique over the Twophase method. Example 1 shows that the two-phase method requires 6 tableaus while our technique requires 3 iterations only, without including the initial one.

\section{4- Illustrative examples}


4.1 Example 1: Consider the following random linear programming problem:

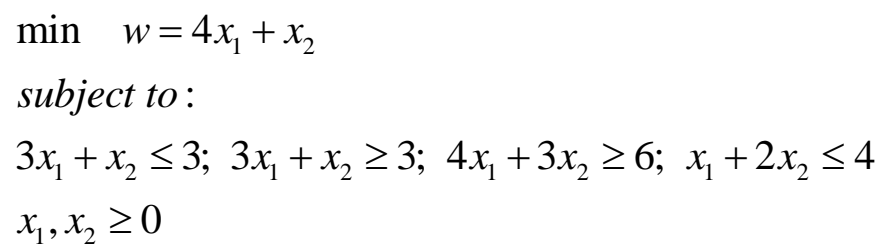

The variables $x_{3}, x_{6}$ and $x_{4}, x_{5}$, below are the slack and surplus variables for the corresponding constraints, respectively. We only need to calculate the corresponding $\cos \theta_{i}$ in the Iteration 0 for every $i=1,2,3$, respectively, as follows:

$$
\begin{aligned}
& \cos \theta_{1}=\# ; \\
& \cos \theta_{2}=\frac{[(-3) \times(-4)+(-1)(-1)]^{2}}{(-3)^{2}+(-1)^{2}}=\frac{169}{10}=16.9 \\
& \cos \theta_{3}=\frac{[(-4) \times(-4)+(-3)(-1)]^{2}}{(-4)^{2}+(-3)^{2}}=\frac{361}{25}=14.44 ; \\
& \cos \theta_{4}=\#
\end{aligned}
$$

The value of $\cos \theta_{2}$ is bigger than that for $\cos \theta_{3}$. We choose $x_{4}$ as the leaving variable. From STEP 2, i.e. the entering variable is calculated as follows:

$\min \left\{\left|b_{i} / a_{i j}\right|: a_{i j}<0\right.$ and $\left.j \in N \quad\right\}=\left\{\left|\frac{-3}{-3}\right|,\left|\frac{-3}{-1}\right|\right\}=1$, therefore the element $x_{1}$ is chosen as the entering variable. The elementary row operations are the employed to construct a new Simplex Tableau (i.e. STEP 3) as shown in Iteration 1 in Table 3. The entire procedure is repeated until all coefficients in Row 0 are non-positive in Iteration 3 and $x_{3}=0, x_{4}=2 / 5, x_{5}=9 / 5$ and $x_{6}=1$ are optimal with $z=17 / 5$ in original the problem.

On the other hand, the two-phase method requires 6 tableaus, as shown in Table 2, without including the initial one.

Table 1The Tableau obtained from the proposed DCSM for Example 1.

\begin{tabular}{lcccccccc}
\hline Iteration & & $x_{1}$ & $x_{2}$ & $x_{3}$ & $x_{4}$ & $x_{5}$ & $x_{6}$ & R.H.S \\
\hline 0 & $\mathrm{Z}$ & -4 & -1 & 0 & 0 & 0 & 0 & 0 \\
& $x_{3}$ & 3 & 1 & 1 & 0 & 0 & 0 & 3 \\
& $x_{4}$ & -3 & -1 & 0 & 1 & 0 & 0 & -3 \\
& $x_{5}$ & -4 & -3 & $\mathbf{0}$ & 0 & 1 & 0 & -6
\end{tabular}




\begin{tabular}{|c|c|c|c|c|c|c|c|c|}
\hline & $x_{6}$ & 1 & 2 & 0 & 0 & 0 & 1 & 4 \\
\hline \multirow[t]{5}{*}{1} & $\mathrm{Z}$ & 0 & 2 & 0 & 0 & -1 & 0 & 6 \\
\hline & $x_{3}$ & 0 & 0 & 1 & 1 & 0 & 0 & 0 \\
\hline & $x_{4}$ & 1 & $1 / 3$ & 0 & $-1 / 3$ & 0 & 0 & 1 \\
\hline & $x_{5}$ & 0 & $-5 / 3$ & 0 & $-4 / 3$ & 1 & 0 & -2 \\
\hline & $x_{6}$ & 0 & $5 / 3$ & 0 & $1 / 3$ & 0 & 1 & 3 \\
\hline \multirow[t]{5}{*}{2} & $\mathrm{Z}$ & 0 & 0 & 0 & $-8 / 3$ & $1 / 5$ & 0 & $18 / 5$ \\
\hline & $x_{3}$ & 0 & 0 & 1 & 1 & 0 & 0 & 0 \\
\hline & $x_{4}$ & 1 & 0 & 0 & $-3 / 5$ & $1 / 5$ & 0 & $3 / 5$ \\
\hline & $x_{5}$ & 0 & 1 & 0 & $4 / 5$ & $-3 / 5$ & 0 & $6 / 5$ \\
\hline & $x_{6}$ & 0 & 0 & 0 & -1 & 1 & 1 & 1 \\
\hline \multirow[t]{5}{*}{3} & $\mathrm{Z}$ & 0 & 0 & 0 & $-7 / 5$ & 0 & $-1 / 5$ & $17 / 5$ \\
\hline & $x_{3}$ & 0 & 0 & 1 & 1 & 0 & 0 & 0 \\
\hline & $x_{4}$ & 1 & 0 & 0 & $-2 / 5$ & 0 & $-1 / 5$ & $2 / 5$ \\
\hline & $x_{5}$ & 0 & 1 & 0 & $1 / 3$ & 0 & $-3 / 5$ & $9 / 5$ \\
\hline & $x_{6}$ & 0 & 0 & 0 & -1 & 1 & 1 & 1 \\
\hline
\end{tabular}

Table 2 The Tableau obtained from the Two-Phase Method for Example 1.

\begin{tabular}{|c|c|c|c|c|c|c|c|c|c|c|}
\hline Iteration & & $x_{1}$ & $x_{2}$ & $x_{3}$ & $x_{4}$ & $x_{5}$ & $R_{1}$ & $R_{2}$ & $x_{6}$ & R.H.S \\
\hline \multirow[t]{5}{*}{ 0 Phase1 } & $Z^{\prime}$ & 0 & 0 & 0 & 0 & 0 & -1 & -1 & 0 & 0 \\
\hline & $x_{5}$ & 3 & 1 & 0 & 0 & 1 & 0 & 0 & 0 & 3 \\
\hline & $R_{1}$ & 3 & 1 & -1 & 0 & 0 & 1 & 0 & 0 & 3 \\
\hline & $R_{2}$ & 4 & 3 & $\mathbf{0}$ & -1 & 0 & 0 & 1 & 0 & 6 \\
\hline & $x_{6}$ & 1 & 2 & $\mathbf{0}$ & 0 & 0 & 0 & 0 & 1 & 4 \\
\hline \multirow[t]{5}{*}{1 Phase 1} & $Z^{\prime}$ & 7 & 4 & -1 & -1 & 0 & 0 & 0 & 0 & 9 \\
\hline & $x_{5}$ & 3 & 1 & 0 & 0 & 1 & 0 & 0 & 0 & 3 \\
\hline & $R_{1}$ & 3 & 1 & -1 & 0 & 0 & 1 & 0 & 0 & 3 \\
\hline & $R_{2}$ & 4 & 3 & 0 & -1 & 0 & 0 & 1 & 0 & 6 \\
\hline & $x_{6}$ & 1 & 2 & 0 & 0 & 0 & 0 & 0 & 1 & 4 \\
\hline \multirow[t]{5}{*}{2 Phase1 } & $Z^{\prime}$ & 0 & 1.67 & -1 & -1 & -2.33 & 0 & 0 & 0 & 2 \\
\hline & $x_{1}$ & 1 & 0.33 & 0 & 0 & 0.33 & 0 & 0 & 0 & 1 \\
\hline & $R_{1}$ & 0 & 0 & -1 & $\mathbf{0}$ & -1 & 1 & 0 & 0 & 0 \\
\hline & $R_{2}$ & 0 & 1.67 & 0 & -1 & -1.33 & 0 & 1 & 0 & 2 \\
\hline & $x_{6}$ & 0 & 0 & 0 & 0 & -0.33 & 0 & 0 & 1 & 3 \\
\hline \multirow[t]{5}{*}{3 Phase 1} & $Z^{\prime}$ & 0 & 0 & -1 & 0 & -1 & 0 & -1 & 0 & 0 \\
\hline & $x_{1}$ & 1 & 0 & 0 & 0.2 & 0.6 & 1 & -0.2 & 0 & 0.6 \\
\hline & $R_{1}$ & 0 & 0 & -1 & 0 & -1 & 0 & 0 & 0 & 0 \\
\hline & $x_{2}$ & 0 & 1 & 0 & -0.6 & -0.8 & 0 & 0.6 & 0 & 1.2 \\
\hline & $x_{6}$ & 0 & 0 & 0 & 1 & 1 & 0 & -1 & 1 & 1 \\
\hline \multirow[t]{4}{*}{4 Phase 2} & $Z^{\prime}$ & 0 & 0 & 0 & 0.2 & 1.6 & blocked & blocked & 0 & 3.6 \\
\hline & $x_{1}$ & 1 & 0 & 0 & 0.2 & 0.6 & 0 & -0.2 & 0 & 0.6 \\
\hline & $R_{1}$ & 0 & 0 & -1 & 0 & -1 & 1 & 0 & 0 & 0 \\
\hline & $x_{2}$ & 0 & 1 & 0 & -0.6 & -0.8 & 0 & 0.6 & 0 & 1.2 \\
\hline
\end{tabular}




$\begin{array}{ccccccccccc} & x_{6} & 0 & 0 & 0 & 1 & 1 & 0 & -1 & 1 & 1 \\ \text { 5 Phase 2 } & \mathrm{Z} & 0 & 0 & -1.6 & 0.2 & 0 & \text { blocked } & \text { blocked } & 0 & 3.6 \\ & x_{1} & 1 & 0 & -0.6 & 0.2 & 0 & 0 & -0.2 & 0 & 0.6 \\ & x_{5} & 0 & 0 & 1 & 0 & 1 & 1 & 0 & 0 & 0 \\ & x_{2} & 0 & 1 & 0.8 & 0.6 & 0 & 0 & 0.6 & 0 & 1.2 \\ & x_{6} & 0 & 0 & -1 & 1 & 0 & 0 & -1 & 1 & 1 \\ & \mathrm{Z} & 0 & 0 & -1.4 & 0 & 0 & \text { blocked } & \text { blocked } & -0.2 & 3.4 \\ & x_{1} & 1 & 0 & -0.4 & 0 & 0 & 0 & -0.2 & -0.2 & 0.4 \\ & x_{5} & 0 & 0 & 1 & 0 & 1 & 1 & 0 & 0 & 0 \\ & x_{2} & 0 & 1 & 0.2 & 1 & 0 & 0 & 0.6 & 0.6 & 1.8 \\ & x_{4} & 0 & 0 & -1 & 0 & 0 & 0 & -1 & 1 & 1\end{array}$

Example 2: Dual Klee-Minty Problem

Consider the following dual Klee-Minty problem of size $n=3$

$$
\begin{aligned}
& \min \quad w=x_{1}+100 x_{2}+10000 x_{3} \\
& \text { subject to: } \\
& x_{1}+20 x_{2}+200 x_{3} \geq 100 ; \quad x_{2}+20 x_{3} \geq 10 ; \quad 4 x_{3} \geq 1, \\
& x_{1}, x_{2}, x_{3} \geq 0
\end{aligned}
$$

The variables $x_{4}, x_{5}, x_{6}$ below are the surplus variables for the corresponding constraints, respectively. We only need to calculate the corresponding $\cos \theta_{i}$ in the Iteration 0 for every $i=1,2,3$, respectively., as follows:

$$
\begin{aligned}
& \cos \theta_{1}=\frac{[(-1) \times(-1)+(-20)(-100)+(-200) \times(-10000)]^{2}}{(-1)^{2}+(-20)^{2}+(-200)^{2}}=\frac{4.0008 \times 10^{12}}{40401}=99027351.81 \\
& \cos \theta_{2}=\frac{[(0) \times(-1)+(-1)(-100)+(-20) \times(-10000)]^{2}}{(0)^{2}+(-1)^{2}+(-20)^{2}}=\frac{4.004001 \times 10^{10}}{401}=99850399 \\
& \cos \theta_{3}=\frac{[(0) \times(-1)+(0)(-100)+(-1) \times(-10000)]^{2}}{(0)^{2}+(0)^{2}+(-1)^{2}}=\frac{10^{8}}{1}=10^{8}
\end{aligned}
$$

The value of $\cos \theta_{3}$ is bigger than that for $\cos \theta_{1}$ and $\cos \theta_{2}$. We choose $x_{6}$ as the leaving variable. From STEP 2, i.e. the entering variable is calculated as follows:

$\min \left\{\left|b_{i} / a_{i j}\right|: a_{i j}<0\right.$ and $\left.j \in N\right\}=\left\{\left|\frac{-100}{-1}\right|,\left|\frac{-100}{-20}\right|,\left|\frac{-100}{-200}\right|\right\}=\frac{1}{2} \quad, \quad$ therefore the element $x_{3}$ is chosen as the entering variable. The elementary row operations are the employed to construct a new Simplex Tableau (i.e. STEP 3) as shown in Iteration 1 in Table 3. The entire procedure is repeated until all coefficients in Row 0 are nonpositive in Iteration 1 and $x_{1}=1, x_{2}=x_{3}=0$ are optimal with $z=10^{4}$ in original the problem. 
Table 3 The Tableau obtained from the proposed DCSM for Example 2.

\begin{tabular}{|c|c|c|c|c|c|c|c|c|}
\hline Iteration & & $x_{1}$ & $x_{2}$ & $x_{3}$ & $x_{4}$ & $x_{5}$ & $x_{6}$ & R.H.S \\
\hline \multirow[t]{4}{*}{0} & $\mathrm{Z}$ & -1 & -10 & -10000 & 0 & 0 & 0 & 0 \\
\hline & $x_{4}$ & -1 & -20 & -200 & 1 & 0 & 0 & -100 \\
\hline & $x_{5}$ & 0 & -1 & -20 & 0 & 1 & 0 & -10 \\
\hline & $x_{6}$ & 0 & 0 & $(-1)$ & 0 & 0 & 1 & -1 \\
\hline \multirow[t]{4}{*}{1} & $\mathrm{Z}$ & -1 & -10 & 0 & 0 & 0 & -10000 & 10000 \\
\hline & $x_{4}$ & -1 & -20 & 0 & 1 & 0 & 200 & 100 \\
\hline & $x_{5}$ & 0 & -1 & 0 & 0 & 1 & 20 & 10 \\
\hline & $x_{3}$ & 0 & 0 & 1 & 0 & 0 & -1 & 1 \\
\hline
\end{tabular}

On the other hand, the two-phase method requires 5 tableaus, as shown in Table 4, without including the initial one.

Table 4 The Tableau obtained from the Two-Phase Method for Example 2.

\begin{tabular}{|c|c|c|c|c|c|c|c|c|c|c|c|}
\hline Iteration & & $x_{1}$ & $x_{2}$ & $x_{3}$ & $x_{4}$ & $x_{5}$ & $x_{6}$ & $R_{1}$ & $R_{2}$ & $R_{3}$ & R.H.S \\
\hline \multirow[t]{4}{*}{0 Phase1 } & $Z^{\prime}$ & 0 & 0 & 0 & 0 & 0 & 0 & -1 & -1 & -1 & 0 \\
\hline & $R_{1}$ & 1 & 20 & 200 & -1 & 0 & 0 & 1 & 0 & 0 & 100 \\
\hline & $R_{2}$ & 0 & -1 & -20 & 0 & -1 & 0 & 0 & 1 & 0 & 10 \\
\hline & $R_{3}$ & 0 & 0 & $(-1)$ & 0 & 0 & -1 & 0 & 0 & 1 & 1 \\
\hline \multirow[t]{4}{*}{1 Phase1 } & $Z^{\prime}$ & 1 & 21 & 221 & -1 & -1 & -1 & 0 & 0 & 0 & 111 \\
\hline & $R_{1}$ & 1 & 20 & 200 & -1 & 0 & 0 & 1 & 0 & 0 & 100 \\
\hline & $R_{2}$ & 0 & 1 & 20 & 0 & -1 & 0 & 0 & 1 & 0 & 10 \\
\hline & $R_{3}$ & 0 & 0 & 1 & 0 & 0 & -1 & 0 & 0 & 1 & 1 \\
\hline \multirow[t]{4}{*}{2 Phase1 } & $Z^{\prime}$ & -0.11 & -1.10 & 0 & 0.11 & -1 & -1 & -1.11 & 0 & 0 & 0.50 \\
\hline & $x_{3}$ & 0.01 & 0.10 & 1 & -0.01 & 0 & 0 & 0.01 & 0 & 0 & 0.50 \\
\hline & $R_{2}$ & -0.10 & -1 & 0 & (0.10) & -1 & 0 & -0.10 & 1 & 0 & 0 \\
\hline & $R_{3}$ & -0.01 & -0.10 & 0 & 0.01 & 0 & -1 & -0.01 & 0 & 1 & 0.50 \\
\hline \multirow[t]{4}{*}{3 Phase 1} & $Z^{\prime}$ & 0 & -0.05 & 0 & 0 & 0.05 & -1 & -1 & -1.05 & 0 & 0.50 \\
\hline & $x_{3}$ & 0 & 0.05 & 1 & 0 & -0.05 & 0 & 0 & 0 & 0 & 0.50 \\
\hline & $x_{4}$ & -1 & -10 & 0 & 1 & -10 & 0 & -1 & 10 & 0 & 0 \\
\hline & $R_{3}$ & 0 & -0.05 & 0 & 0 & 0.05 & -1 & 0 & -0.05 & 1 & 0.50 \\
\hline \multirow[t]{4}{*}{4 Phase1 } & $Z^{\prime}$ & 0 & 0 & 0 & 0 & 0 & 0 & -1 & -1 & -1 & 0 \\
\hline & $x_{3}$ & 0 & 0 & 1 & 0 & 0 & -1 & 0 & 0 & 1 & 1 \\
\hline & $x_{4}$ & -1 & -20 & 0 & 1 & 0 & -200 & -1 & 0 & 200 & 100 \\
\hline & $R_{3}$ & 0 & -1 & 0 & 0 & 1 & -20 & 0 & -1 & 20 & 10 \\
\hline \multirow[t]{4}{*}{5 Phase 2} & $Z^{\prime}$ & -1 & -100 & 0 & 0 & 0 & $-10^{4}$ & blocked & blocked & blocked & 10000 \\
\hline & $x_{3}$ & 0 & 0 & 1 & 0 & 0 & -1 & 0 & 0 & 1 & 1 \\
\hline & $x_{4}$ & -1 & -20 & 0 & 1 & 0 & -200 & -1 & 0 & 200 & 100 \\
\hline & $x_{5}$ & 0 & -1 & 0 & 0 & 1 & -20 & 0 & -1 & 20 & 10 \\
\hline
\end{tabular}

\section{Computational Experiments}

In this section, we present the computational results of dual cosine simplex algorithm (DDCA) and two - phase method for dual Klee-Minty and dual Clauser classes of problems. We compare the number of iterations of dual cosine simplex 
algorithm (DDCA) with two - phases method. In each test problem, we used different tolerances in order to get the smaller number of iterations with the exact optimum solution. For this comparison, we chose the two phase method [12-15] for the problems contain " $\geq$ " constraints and/or equality constraints.

The programming language used was MATLAB v7.01 SP2 with default options. All codes were run under 64-bit Window 8.1 Operating System having Core(TM)i5 CPU M $460 @ 2.53 \mathrm{GHz}, 4.00 \mathrm{~GB}$ of memory.

Table 5 The Tableau obtained from the dual cosine, Two-Phases and dual simplex.

\begin{tabular}{|c|c|c|c|c|}
\cline { 2 - 5 } \multicolumn{1}{c|}{} & \multicolumn{2}{c|}{ Dual Klee-Minty problem } & \multicolumn{2}{c|}{ Dual Clauser problem } \\
\hline Size & $\begin{array}{c}\text { Dual cosine } \\
\text { DDCA }\end{array}$ & $\begin{array}{c}\text { Two phase } \\
\text { method }\end{array}$ & $\begin{array}{c}\text { Dual cosine } \\
\text { DDCA }\end{array}$ & $\begin{array}{c}\text { Two phase } \\
\text { method }\end{array}$ \\
\hline 1 & 2 & 1 & 4 & 3 \\
\hline 2 & 2 & 3 & 4 & 4 \\
\hline 3 & 2 & 4 & 4 & 5 \\
\hline 4 & 2 & 5 & 4 & 7 \\
\hline 5 & 2 & 6 & 4 & 9 \\
\hline 6 & 2 & 7 & 4 & 11 \\
\hline 7 & 2 & 8 & 4 & 13 \\
\hline 8 & 2 & 9 & 4 & 15 \\
\hline 9 & 2 & 10 & 4 & 17 \\
\hline 10 & 2 & 11 & 4 & 19 \\
\hline
\end{tabular}

From Table 5, the contribution of the proposed algorithm is to solve KleeMinty problem and Clausen problem with 2 and 4 iterations, respectively, while the simplex method with two phase method spends $O(n)$ iterations for these problems.

Table 6 characterizes 33 NETLIB test problems [16] were used in comparison to test the performance of the algorithms. We transformed the variables ( consist of bounds or are free without limitation) into constraints to keep the algorithms simple. We used LINGO to test the accuracy of the answers obtained using our algorithms.

Table 6 Properties of 33 NETLIB problems

\begin{tabular}{|c|c|c|c|c|c|c|c|c|}
\hline $\begin{array}{l}\text { Problem } \\
\text { name }\end{array}$ & $\begin{array}{l}\begin{array}{l}\text { Number } \\
\text { of } \\
\text { onzeros }\end{array} \\
\end{array}$ & Density & $\begin{array}{l}\text { New } \\
\text { number of } \\
\text { constraints }\end{array}$ & $\begin{array}{l}\text { New } \\
\text { number of } \\
\text { variables } \\
\end{array}$ & $\begin{array}{l}\begin{array}{l}\text { Number } \\
\text { of } \\
\text { variables }\end{array} \\
\end{array}$ & $\begin{array}{l}\text { Number of } \\
" \leq " \\
\text { constrains }\end{array}$ & $\begin{array}{l}\text { Number } \\
\text { of } " \geq " \\
\text { constrains }\end{array}$ & $\begin{array}{c}\text { Number } \\
\text { of " = " } \\
\text { constrains }\end{array}$ \\
\hline adlittle & 465 & 0.0856 & 56 & 97 & 97 & 40 & 1 & 15 \\
\hline afiro & 88 & 0.10185 & 27 & 32 & 32 & 19 & 0 & 8 \\
\hline bandm & 2659 & 0.01847 & 305 & 472 & 472 & 0 & 0 & 305 \\
\hline beaconfd & 3476 & 0.07669 & 173 & 262 & 262 & 33 & 0 & 140 \\
\hline brandy & 2150 & 0.03925 & 220 & 249 & 249 & 54 & 0 & 166 \\
\hline etamacro & 2489 & 0.00547 & 400 & 688 & 688 & 183 & 125 & 354 \\
\hline
\end{tabular}




\begin{tabular}{|c|c|c|c|c|c|c|c|c|}
\hline fit1d & 14,430 & 0.0134 & 24 & 1026 & 1026 & 1038 & 11 & 1 \\
\hline fit1p & 10,894 & 0.00633 & 627 & 1677 & 1677 & 399 & 0 & 627 \\
\hline grow15 & 5665 & 0.00976 & 300 & 645 & 645 & 600 & 0 & 300 \\
\hline grow22 & 8318 & 0.00666 & 440 & 946 & 946 & 880 & 0 & 440 \\
\hline grow7 & 2633 & 0.02083 & 140 & 301 & 301 & 280 & 0 & 140 \\
\hline kb2 & 291 & 0.13649 & 43 & 41 & 41 & 21 & 15 & 16 \\
\hline lotfi & 1086 & 0.02305 & 153 & 308 & 308 & 42 & 16 & 95 \\
\hline recipelp & 752 & 0.0198 & 91 & 180 & 180 & 77 & 43 & 91 \\
\hline sc105 & 281 & 0.02598 & 105 & 103 & 103 & 60 & 0 & 45 \\
\hline sc205 & 552 & 0.01326 & 205 & 203 & 203 & 114 & 0 & 91 \\
\hline sc50a & 131 & 0.05458 & 50 & 48 & 48 & 30 & 0 & 20 \\
\hline sc50b & 119 & 0.04958 & 50 & 48 & 48 & 30 & 0 & 20 \\
\hline scagr25 & 2029 & 0.00862 & 471 & 500 & 500 & 146 & 25 & 300 \\
\hline scagr7 & 553 & 0.03062 & 129 & 140 & 140 & 38 & 7 & 84 \\
\hline scfxm1 & 2612 & 0.01732 & 330 & 457 & 457 & 143 & 0 & 187 \\
\hline scfxm2 & 5229 & 0.00867 & 660 & 914 & 914 & 286 & 0 & 374 \\
\hline scfxm3 & 7846 & 0.00578 & 990 & 1371 & 1371 & 429 & 0 & 561 \\
\hline $\operatorname{scsd} 1$ & 3148 & 0.05379 & 77 & 760 & 760 & 0 & 0 & 77 \\
\hline $\operatorname{scsd6}$ & 5666 & 0.02855 & 147 & 1350 & 1350 & 0 & 0 & 147 \\
\hline sctap1 & 2052 & 0.01425 & 300 & 480 & 480 & 0 & 180 & 120 \\
\hline share1b & 1182 & 0.0449 & 117 & 225 & 225 & 28 & 0 & 89 \\
\hline share2b & 730 & 0.09626 & 96 & 79 & 79 & 83 & 0 & 13 \\
\hline shell & 4900 & 0.00303 & 536 & 1775 & 1775 & 119 & 9 & 784 \\
\hline ship04l & 8450 & 0.00992 & 402 & 2118 & 2118 & 40 & 8 & 354 \\
\hline ship04s & 5810 & 0.00991 & 402 & 1458 & 1458 & 40 & 8 & 354 \\
\hline stair & 3857 & 0.0186 & 356 & 467 & 467 & 153 & 0 & 698 \\
\hline stocfor1 & 474 & 0.0365 & 117 & 111 & 111 & 48 & 6 & 63 \\
\hline Sum & 111,017 & 1.09377 & 8539 & 19,531 & 19,531 & 5453 & 454 & 7079 \\
\hline Average & 3364.152 & 0.03315 & 258.758 & 591.849 & 591.849 & 165.242 & 13.7576 & 214.515 \\
\hline Max & 14,430 & 0.13649 & 990 & 2118 & 2118 & 1038 & 180 & 784 \\
\hline Min & 88 & 0.00303 & 24 & 32 & 32 & 0 & 0 & 1 \\
\hline
\end{tabular}

Tables 6 contains 6 categories of the problems according to the variable numbers range as 30-99, 100-500, 501-999, 1000-1500, 1501-1999 and over 2000 were 6, 15, 5, 4, 2 and 1, respectively. Table 6 contains the largest nonzero number, density, number of variables (after transferring sign constraints), number of constraints (after transferring sign constraints), " $\leq$ " constraint number, " $\geq$ " constraint number, and "=" constraint number.

Table 7 Comparison between the proposed DDCA and two phase method

\begin{tabular}{|c|c|c|c|c|c|c|c|c|c|}
\hline \multirow{3}{*}{$\begin{array}{l}\text { Problem } \\
\text { name }\end{array}$} & \multicolumn{6}{|c|}{ Iterations number } & \multirow{2}{*}{\multicolumn{3}{|c|}{$\begin{array}{c}\text { Difference in iteration number } \\
\text { Simplex - DCA }\end{array}$}} \\
\hline & \multicolumn{3}{|c|}{ DCA } & \multicolumn{3}{|c|}{ Simplex } & & & \\
\hline & $\begin{array}{l}\text { Phase } \\
\text { I }\end{array}$ & $\begin{array}{l}\text { Phase } \\
\text { I I }\end{array}$ & $\begin{array}{l}\text { Phase } \\
\text { I\&II }\end{array}$ & $\begin{array}{l}\text { Phase } \\
\text { I }\end{array}$ & $\begin{array}{l}\text { Phase } \\
\text { I I }\end{array}$ & $\begin{array}{l}\text { Phase } \\
\text { I\&II }\end{array}$ & $\begin{array}{l}\text { Phase } \\
\text { I }\end{array}$ & $\begin{array}{l}\text { Phase } \\
\text { I I }\end{array}$ & $\begin{array}{l}\text { Phase } \\
\text { I\&II }\end{array}$ \\
\hline adlittle & 21 & 99 & 120 & 38 & 100 & 138 & 17 & 1 & 18 \\
\hline afiro & 6 & 7 & 13 & 10 & 7 & 17 & 4 & 0 & 4 \\
\hline
\end{tabular}




\begin{tabular}{|c|c|c|c|c|c|c|c|c|c|}
\hline bandm & 828 & 323 & 1151 & 1042 & 242 & 1284 & 214 & -81 & 133 \\
\hline beaconfd & 132 & 17 & 149 & 154 & 37 & 191 & 22 & 20 & 42 \\
\hline brandy & 731 & 82 & 813 & 521 & 71 & 592 & -210 & -11 & -221 \\
\hline etamacro & 940 & 355 & 1295 & 944 & 423 & 1367 & 4 & 68 & 72 \\
\hline fit1d & 52 & 1664 & 1716 & 94 & 1355 & 1449 & 42 & -309 & -267 \\
\hline fit1p & 820 & 2288 & 3108 & 1441 & 1358 & 2799 & 621 & -930 & -309 \\
\hline grow15 & 285 & 205 & 490 & 303 & 485 & 788 & 18 & 280 & 298 \\
\hline grow22 & 425 & 245 & 670 & 443 & 704 & 1147 & 18 & 459 & 477 \\
\hline grow7 & 131 & 78 & 209 & 143 & 168 & 311 & 12 & 90 & 102 \\
\hline kb2 & 74 & 25 & 99 & 397 & 38 & 435 & 323 & 13 & 336 \\
\hline lotfi & 208 & 164 & 372 & 126 & 77 & 203 & -82 & -87 & -169 \\
\hline recipelp & 300 & 6 & 306 & 299 & 28 & 327 & -1 & 22 & 21 \\
\hline sc105 & 54 & 46 & 100 & 64 & 42 & 106 & 10 & -4 & 6 \\
\hline sc205 & 118 & 110 & 228 & 128 & 115 & 243 & 10 & 5 & 15 \\
\hline sc50a & 24 & 20 & 44 & 29 & 23 & 52 & 5 & 3 & 8 \\
\hline sc50b & 32 & 14 & 46 & 37 & 21 & 58 & 5 & 7 & 12 \\
\hline scagr25 & 503 & 869 & 1372 & 639 & 218 & 857 & 136 & -651 & -515 \\
\hline scagr7 & 126 & 85 & 211 & 159 & 45 & 204 & 33 & -40 & -7 \\
\hline scfxm1 & 753 & 252 & 1005 & 802 & 211 & 1013 & 49 & -41 & 8 \\
\hline $\operatorname{scfxm2}$ & 1592 & 322 & 1914 & 1478 & 386 & 1864 & -114 & 64 & -50 \\
\hline scfxm3 & 1947 & 490 & 2437 & 2324 & 591 & 2915 & 377 & 1 & 378 \\
\hline scsd1 & 90 & 200 & 290 & 139 & 206 & 345 & 49 & 6 & 55 \\
\hline $\operatorname{scsd6}$ & 216 & 184 & 400 & 170 & 447 & 617 & -46 & 263 & 217 \\
\hline sctap1 & 453 & 161 & 614 & 705 & 163 & 868 & 252 & 2 & 254 \\
\hline share1b & 352 & 224 & 576 & 363 & 158 & 521 & 11 & -66 & -55 \\
\hline share2b & 125 & 50 & 175 & 112 & 27 & 139 & -13 & -23 & -36 \\
\hline shell & 795 & 264 & 1059 & 843 & 209 & 1052 & 48 & -55 & -7 \\
\hline ship04l & 700 & 143 & 843 & 728 & 78 & 806 & 28 & -65 & -37 \\
\hline ship04s & 488 & 106 & 594 & 499 & 58 & 557 & 11 & -48 & -37 \\
\hline stair & 1019 & 323 & 1342 & 1203 & 265 & 1468 & 184 & -58 & 126 \\
\hline stocfor1 & 81 & 12 & 93 & 90 & 29 & 119 & 9 & 17 & 26 \\
\hline Sum & 14421 & 9433 & 23854 & 16467 & 8385 & 24852 & & & \\
\hline Average & 437 & 285.848 & 722.848 & 499 & 254.091 & 753.091 & & & \\
\hline Max & 1947 & 2288 & 3108 & 2324 & 1358 & 2915 & & & \\
\hline Min & 6 & 6 & 13 & 10 & 7 & 17 & & & \\
\hline
\end{tabular}

In general, from Table 7, the contribution of the proposed algorithm is that DDCA is generally better than two phase method ( 22 problems vs. 11 problems). The details of our results as the following:

a) Six problems with the variable numbers 30-99:

DDCA is better than two phase method (5 problems vs. one problem)

b) Fifteen problems with the variable numbers 100-500:

DDCA is better than two phase method (10 problems vs. 5 problems)

c) Five problems with the variable numbers 501-999: 
DDCA is better than two phase method (4 problems vs. one problem)

d) Four problems with the variable numbers 1000-1500:

DDCA and two phase methods are equal (2 problems vs. 2 problems)

e) Two problems with the variable numbers 1501-1999:

Two phase method is better than DDCA ( 0 problems vs. 2 problems)

f) One problem with the variable numbers over 2000:

Two phase method is better than DDCA (0 problems vs. 1 problem)

\section{Conclusions}

We proposed a dual version of the direct cosine simplex algorithm (DDCA) for general linear problems. Unlike the two-phase and the big-M methods, our technique does not involve artificial variables. Our technique solved the dual KleeMinty problem in two iterations and solved the dual Clausen's problem in four iterations. The utility of the proposed method is evident from the extensive computational results on test problems adapted from NETLIB. Preliminary results indicate that this dual direct cosine simplex algorithm (DDCA) reduces the number of iterations of two-phase method.

\section{References}

[1] G.B. Dantzig, Maximization of a linear function of variables subject to linear inequalities, in: T.C. Koopmans (Ed.), Activity Analysis of production and Allocation, John Wiley, NY, 1951, pp. 339-347.

[2] G.B. Dantzig, Linear Programming and Extensions, Princeton Univ. Press, Princeton, NJ, 1963.

[3] M.S. Bazaraa, J.J. Jarvis, H.D. Sherali, Linear Programming and Network Flows, third ed., John Wiley, NY, 2004.

[4] K. Paparrizos, An exterior point simplex algorithm for general linear problems, Annals of Operation Research 32 (1993) 497-508.

[5] E. S. Badr, K. Paparrizos, N. Samaras, and A. Sifaleras (2005), On the Basis Inverse of the Exterior Point Simplex Algorithm, in Proc. of the 17th National Conference of Hellenic Operational Research Society (HELORS), 16-18 June, Rio, Greece, pp. 677-687.

[6] E.S. Badr, K. Paparrizos, Baloukas Thanasis and G. Varkas (2006), Some computational results on the efficiency of an exterior point algorithm, in Proc. of the 18th National Conference of Hellenic Operational Research Society (HELORS), 15-17 June, Rio, Greece, pp. 1103-1115 
[7] M. Tipawanna and K. Sinapiromsaran (2013), Max-out-in pivot rule with Dantzig's safeguarding rule for the simplex method, 2nd International Conference on Mathematical Modeling in Physical Sciences

[8] W.-C. Yeh and H.W. Corley, A simple direct cosine simplex algorithm, Applied Mathematics and Computing. 214 (2009) 178-186.

[9] V. Klee, G. Minty, How good is the simplex algorithm?, in: O. Shisha (Ed.), Inequalities-III, Academic Press, NY, 1972, pp. 159-175.

[10] J.Clausen. A tutorial note on the complexity of the simplex algorithm. Technicla Report NR79/16, DIKU, Copenhagen, Denmark, 1979.

[11] K. G. Murty. Linear Programming. John Wiley and Sons, New Yourk, 1983.

[12] R. Vanderbei, Linear Programming: Foundations and Extensions, second ed., Kluwer Academic Publishers, Boston, 2001.

[13] P.E.Gill,W.Murray,M.H.Wright,PracticalOptimization,AcademicPress,NY,1981.

[14] F.S. Hiller, G.J. Lieberman, Introduction to Operations Research, sixth ed., McGraw-Hill, NY, 1995.

[15] M.S. Bazaraa, J.J. Jarvis, H.D. Sherali, Linear Programming and Network Flows, third ed., John Wiley, NY, 2004.

[16] <http://www.netlib.org/lp/data> .

[17] Elsayed M. Badr, Mahmoud I. Moussa in Wireless Networks (2019), An upper bound of radio $k$-coloring problem and its integer linear programming model, First Online: 18 March 2019.

[18] Badr, E.;Aloufi,K.A Robot's Response Acceleration Using the Metric Dimension Problem. Preprints 2019, 2019110194 (doi: 10.20944/preprints201911.0194.v1). 